\title{
Un enfoque de gobernanza aplicado al análisis de una investigación sobre trabajadores cesantes en Medellín, Colombia
}

\section{A governance approach applied to analysing research into unemployed workers in the city of Medellin in Colombia}

Álvaro Cardona, Emmanuel Nieto y Luz M. Mejía

Facultad Nacional de Salud Pública. Universidad de Antioquia. Medellín, Colombia. alvarocardona66@hotmail.com, emmanuel@guajiros.udea.edu.co, luzmmejia@guajiros.udea.edu.co

Recibido 15 Julio 2009/Enviado para Modificación 16 Diciembre 2009/Aceptado 12 Enero 2010

\section{RESUMEN}

Objetivo Realizar un ejercicio académico de aplicación de las categorías analíticas del enfoque de gobernanza desarrollado por Marc Hufty para comprender el pro-ceso de relacionamiento de actores sociales en un proyecto de investigación e intervención que estudió las condiciones socioeconómicas y de aseguramiento en salud de los trabajadores cesantes-TC en la ciudad de Medellín-Colombia en los años 2004 y 2007 y que incorporó la propuesta de una política pública para garanti-zar la continuidad de su aseguramiento en salud.

Metodología Se trató del examen como caso de un proceso de investigación e intervención, en el que los investigadores fueron uno de los actores involucrados. La caracterización de los actores involucrados incluyó: nivel de inser-ción/ involucramiento en el problema; poder de decisión en la política pública pro-puesta; características, fuerza y dinámica de sus percepciones, expectativas y pro-puestas sobre el problema del desempleo y del aseguramiento en salud cuando alguien queda cesante; características de su interacción con otros actores.

Resultados Se encontró que las dimensiones analíticas propuestas por Hufty (acto-res, normas sociales, puntos nodales y procesos) resultaron útiles para describir y comprender el proceso de interacción de actores sociales involucrados en la inves-tigación y la propuesta de intervención que sirvió como caso de estudio. Conclusiones El marco analítico de gobernanza propuesto por Hufty fue útil para entender cómo interactuaron los sujetos sociales, cuáles fueron las normas acogi-das para interactuar, cuáles nodos de actuación fueron más importantes y los pro-gresos alcanzados durante el proceso.

Palabras Clave: Desempleo, políticas públicas, seguridad social, seguro de salud, Colombia (fuente: DeCS, BIREME). 


\section{ABSTRACT}

Objective Performing an academic exercise aimed at applying the analytical catego-ries from the governance approach developed by Marc Hufty et al., to understand social actors' relationships in an investigation and intervention project studying so-cioeconomic conditions and seeking to guarantee health insurance continuity for those workers who had lost their work in the city of Medellin, Colombia, from 2004 to 2007.

Methodology A process of investigation and intervention was examined as a casestudy in which researchers were one of the actors so involved. Characterising stake-holders included: their level of inclusion/involvement in the problem; their power for influencing public policy proposals; their perceptions and proposals' characteristics, power and dynamics regarding the problem of unemployment and health insurance when someone has lost her/his work; and the characteristics of their interaction with other actors.

Results The results showed that the four analytical dimensions proposed by Hufty (actors, social norms, nodal points and processes) were useful for describing and understanding the interaction of the actors involved in the research and intervention proposal being analysed here (i.e. the case-study).

Conclusions it was concluded that the analytical governance framework proposed by Hufty was useful for understanding how the social subjects interacted; these were the rules which were taken for describing their interaction, being the most important nodes for interaction and progresses achieved whilst implementing the intervention proposal.

Key Words: Governance, government, unemployment, public policy, social security, insurance, health, Colombia (source: $M e S H, N L M$ ).

a incorporación del concepto de "Gobernanza" en los estudios sociales y políticos se remonta a los finales de la década de 1980, siendo en nuestros días de uso común en la academia y en los medios gubernamentales $(1,2)$.

Para Nick Bernard, este concepto "...insiste en el reparto del poder, la diversidad y la descentralización, los instrumentos flexibles y la reafirmación de la primacía de los procesos políticos sobre los legales" (3).

Según Tim Plumptre y John Graham, "Gobernanza se refiere a cómo interactúan los gobiernos y otras organizaciones sociales, cómo se relacionan con los ciudadanos y cómo se toman las decisiones en un mundo crecientemente complejo" (1). Agregan: "nos parece claro que la gobernanza involucra las interacciones entre estructuras, procesos y tradiciones que determinan como se ejerce el poder, como se toman las decisiones y como los ciudadanos $u$ otros actores interesados manifiestan sus opiniones. Fundamentalmente, se refiere al poder, las relaciones y la rendición de cuentas: quién tiene influencia, quién decide y cómo los tomadores de decisiones se hacen responsables" (1). Según ellos mismos, "gobernanza no se refiere sólo a donde ir, sino que también se refiere a quiénes deben ser involucrados en las decisiones y en que calidad" (1). 
En opinión de Marc Hufty, el concepto de "Gobernanza" ha atendido a la comprensión del hecho de que en el proceso de toma de decisiones sobre asuntos de importancia pública está involucrada la participación de actores sociales diferentes del gobierno. En consecuencia, este concepto busca esclarecer la manera como los actores interactúan en esas circunstancias (2).

A pesar de estas importantes coincidencias, emergen diferentes enfoques cuando se discute sobre la formulación de un modelo ideal de gobernanza para cualesquiera sociedad. Hufty adopta un abordaje que llama no normativo de la Gobernanza, de acuerdo con el cual no cabría pensar en una "buena Gobernanza", sino en múltiples formas de "Gobernanza" según las condiciones particulares de existencia de los actores sociales, la manera específica como se relacionan para resolver asuntos públicos y las especificidades del contexto histórico-cultural de cada sociedad (2). En sus propias palabras, "Existen entonces numerosas formas de gobernanza, en .la historia, pero aun en el presente. Estas diferentes formas pueden ser objeto de un análisis y una interpretación. Ellas pueden ser descritas y desagregadas en sus elementos constitutivos a fin de comprender su funcionamiento" (2) Este enfoque contrasta con la visión de Plumptre y Graham, para quienes la finalidad ideal de los actores sociales es encontrar un modelo de "buena Gobernanza". (1)

La preocupación central de Hufty es la utilización del concepto como herramienta para aprehender las intimidades de las relaciones entre actores. $\mathrm{Su}$ propósito es transformar el concepto de gobernanza en un "concepto útil para una mejor comprensión de la realidad" (2) y consecuencialmente para promover la acción de los actores y el cambio de esa realidad. Para ello ha propuesto lo que llama "un marco analítico de gobernanza", definido como "un conjunto coherente de modelos (representaciones esquemáticas de una situación) asociado a una metodología que permita el pasaje entre el marco conceptual y la observación empírica" (2).

$\mathrm{Al}$ efecto, formula que el estudio de la gobernanza puede realizarse a través de la disociación en elementos constitutivos observables y de la caracterización de las interacciones y sus consecuencias y propone cuatro categorías o "dimensiones analíticas" con su correspondiente definición: los actores, las normas sociales, los puntos nodales y los procesos. "Los actores son individuos o grupos. Su acción colectiva (acuerdos o decisiones) conduce a la formulación de normas (o reglas de juego / o decisiones) que orientan el comportamiento de los actores y son modificadas por la acción colectiva. La acción colectiva resulta de la interacción/transacción entre los actores, ya sea conflictiva o cooperativa, de los acuerdos o las decisiones que se toman y de su aplicación. (...) Puntos nodales [son] espacios físicos o virtuales (...) en donde convergen varios procesos, actores y normas, que producen efectos, aisladamente o en interacción con otros, sobre la variable dependiente estudiada. Los procesos son sucesiones de estados por los cuales pasan la interrelación entre actores, normas y puntos nodales" (2). 
Según sus proponentes, estas dimensiones analíticas permiten identificar y caracterizar las formas específicas como se expresa el proceso de gobernanza en el abordaje de cada problema social sometido a análisis y extraer hipótesis sobre su relación con la expresión concreta del problema analizado, que asume la forma de variable dependiente.

En este artículo se presenta un ejercicio de aplicación de las categorías analíticas del enfoque de gobernanza desarrollado por Hufty (2) para comprender el interrelacionamiento de actores sociales propiciado por una investigación que tuvo por objetivos establecer las condiciones socioeconómicas y de aseguramiento en salud de los trabajadores que perdieron su empleo (trabajadores cesantes-TC) en la ciudad de Medellín-Colombia en los años 2004 y 2007 e incluyó la propuesta de una política de intervención que garantice la continuidad de su aseguramiento $(4,5)$.

La política de intervención consiste en la creación de un "Fondo de subsidio y crédito reembolsable" que subsidiaría a los TC, por un periodo máximo de seis meses de cesantía, la tercera parte de los aportes al Régimen Contributivo del Sistema General de Seguridad Social en Salud (SGSSS), al tiempo que les concedería un crédito correspondiente a las restantes dos terceras partes. El monto del préstamo deberá amortizarse por los TC cuando vuelvan a emplearse. La evaluación ex ante de la sostenibilidad financiera del Fondo se hizo mediante consultoría con expertos. La política se justifica en el hecho de que para 2004 el $36,7 \%$ de los TC de la ciudad de Medellín quedó sin aseguramiento en salud (4). Un nuevo estudio, pendiente de publicación, ha mostrado que para 2007, esta cifra ascendió a 46,5\%.

\section{MÉTODOS}

Se trata del examen como caso de un proceso de investigación e intervención, en el que los investigadores son uno de los actores involucrados al haber generado el proceso social de interacción alrededor de la propuesta de intervención. Otros actores intervinientes fueron: trabajadores cesantes-TC, líderes políticos de la ciudad, Entidades Promotoras de Salud (EPS), Cajas de Compensación Familiar (CCF), representantes de empleadores, líderes de organizaciones sindicales y medios de comunicación.

Las preguntas problematizadoras que se le hicieron al modelo propuesto por Hufty fueron: a. ¿Cuál es su utilidad principal en los análisis de los procesos de formación de políticas públicas en salud?; b. ¿Qué aportes puede generar a los 
investigadores de políticas públicas en salud? y; c. ¿De qué manera este enfoque pudiera complementarse con otros modelos utilizados por la salud pública?

El referente empírico de los resultados que se presentan en este artículo lo constituyen las actitudes, manifestaciones y compromisos expresados por los actores en las distintas actividades impulsadas y/o enmarcadas en nuestro proyecto de investigación e intervención, tales como entrevistas semi-estructuradas individuales y grupales, talleres de interacción de acuerdo con la metodología de Mapeo de Alcances (6), interacción con medios de comunicación y reuniones con líderes políticos de la ciudad.

La caracterización de los actores involucrados incluye: nivel de inserción/ involucramiento en el problema; poder que tienen para definir la política pública propuesta; características, fuerza y dinámica de sus percepciones; expectativas y propuestas sobre el problema del desempleo y del aseguramiento en salud cuando alguien queda cesante; características de su interacción con otros actores.

\section{Contexto del Caso Estudiado}

El Sistema General de Seguridad Social en Salud en Colombia. La normatividad colombiana sobre seguridad social en salud se caracteriza por establecer diferentes tipos de aseguramiento en función de la vinculación al mercado laboral (7). Las personas con vinculación laboral y/o capacidad de cotizar se afilian a través del Régimen Contributivo y tienen derecho a los servicios definidos en el Plan Obligatorio de Salud (POS-C) (8). Quienes no cumplen estas condiciones pueden afiliarse al Régimen Subsidiado, de acuerdo con los cupos habilitados por los recursos financieros dispuestos por el Estado para este fin y complementados con recursos solidarios provenientes de un porcentaje de las cotizaciones del régimen contributivo. Este régimen garantiza los servicios definidos en el Plan Obligatorio de Salud Subsidiado (POS-S) (9,10), con menor cantidad de servicios que el POS-C. En 2008 la cobertura poblacional de aseguramiento en los regímenes contributivo y subsidiado era respectivamente de $39,7 \%$ y $39,7 \%$ (11).

El sistema contempla que las personas no afiliadas a alguno de estos regímenes reciban servicios asistenciales con cargo a los municipios o departamentos, según sea el nivel de complejidad de los mismos (12). El acceso está supeditado a la disponibilidad de recursos financieros que el gobierno nacional transfiere para este fin a estos entes territoriales. 
El desempleo en Colombia. En el periodo 1996-2002 el crecimiento promedio anual del PIB colombiano fue de solo $1,1 \%$. En junio de 2000 el desempleo fue de 20,4\% en las principales siete áreas metropolitanas (Bogotá, Medellín, Cali, Barranquilla, Manizales, Bucaramanga, Pasto). En Medellín alcanzó el $20,8 \%(13,14)$.

En los años 2003-2004 se inició un periodo de recuperación de la actividad productiva, que alcanzó el nivel más alto en 2007 con un crecimiento del PIB de 7,5\%. La tasa de desempleo descendió al 11,6\% en las 13 principales áreas metropolitanas en junio de ese año. La ciudad de Medellín sólo logró abatir esta tasa hasta $12,3 \%$, que representaba cerca de 185.000 desempleados, de los cuales el $82 \%$ correspondía a TC $(15,16)$.

Con el inicio de la recesión mundial de 2008 la economía colombiana redujo el crecimiento del PIB en ese año a 2,5 \% (17). En el mes de mayo de 2009 el desempleo nacional había ascendido a 11,7 \% y en Medellín a $15 \%$ (18).

Dado que las alternativas de aseguramiento en salud están asociadas al tipo de vinculación al mercado laboral, se comprende que las mayores tasas de desempleo tienden a generar dificultades en la cobertura de aseguramiento y a vulnerar el derecho al acceso a servicios de salud de los TC y sus familias.

\section{RESULTADOS}

Los actores: características relevantes e interacción

El modelo analítico de gobernanza permitió la siguiente caracterización de los actores involucrados en la propuesta de establecer una política pública que garantice al aseguramiento en salud de los TC:

Los Trabajadores Cesantes TC. Serían, con sus familias, los beneficiarios directos de la política pública propuesta, en tanto son los principales afectados por el desempleo y su consecuente pérdida de aseguramiento en salud. Con su participación en el proceso lograron constituir la primera Asociación de Trabajadores Cesantes del país, como instancia organizativa para mediar su interacción con otros actores.

En sus actuaciones se percibió una alta valoración de la propuesta por el relevamiento que hace del derecho a la salud. Sin embargo, muchos TC mantienen gran desconfianza en las instituciones del Estado y dudan de su 
propia posibilidad de incidir en políticas públicas que los beneficien. Estas percepciones poco esperanzadoras están relacionadas con la desmotivación derivada de su condición de desempleados. La dependencia económica de otros miembros de la familia a la que frecuentemente quedan sometidos, es uno de los aspectos que mayor frustración les produce.

La pobre tradición organizativa, la precariedad de sus recursos económicos y la desconfianza en su capacidad para incidir en las políticas públicas, determinan que tengan actualmente poca posibilidad de incidir en la toma de decisiones que alivien su situación de no aseguramiento en salud. Esas mismas razones hacen que la interacción con otros actores sea frecuentemente intermediada por actores con mayor reconocimiento social. Este ha sido el caso de la intermediación del grupo de investigación, que ha propiciado diversas modalidades de inter-relacionamiento de actores, en las que los líderes de los TC han debatido y formulado sus demandas.

Los agrupamientos políticos de la ciudad. Son un actor bastante heterogéneo por la multiplicidad de organizaciones políticas que representan. Su participación en instancias decisorias para la formación de políticas públicas en la ciudad les confiere poder para influir en la decisión que se adopte con respecto a la política pública propuesta.

Su apoyo mayoritario a las políticas del gobierno nacional y su escasa apertura para considerar propuestas alternativas de protección social en salud, hacen que su involucramiento en el tema del aseguramiento en salud de los TC sea marginal. Sin embargo, algunos concejales de la municipalidad, en razón de su posicionamiento crítico frente al marco normativo del SGSSS, han abanderado la propuesta de esta política pública.

El soporte conceptual de su actitud ha sido que el derecho a la salud debe ser garantizado por el Estado y la sociedad, independientemente de la condición de estar empleado o no. Así mismo, le atribuyen un valor especial a la protección de la salud de la población como requisito para el desarrollo social, la productividad y competitividad de la ciudad.

Las entidades administradoras de planes de beneficios del SGSSS de Medellín. En tanto que de su actividad han derivado grandes beneficios económicos, no muestran interés en modificaciones significativas al modelo de aseguramiento en salud. Sin embargo, sus expectativas económicas los disponen a cooperar en propuestas que extiendan la cobertura del aseguramiento. 
$\mathrm{Su}$ interacción en el proceso alrededor de la propuesta del grupo de investigación ha sido bastante circunstancial y episódica. No obstante, han manifestado su apoyo a la iniciativa de garantizar el aseguramiento en salud de los TC, argumentando la utilidad que tendría como mecanismo de eficiencia administrativa y financiera al disminuir la alta rotación de los afiliados en el sistema por la pérdida del empleo, al tiempo que ayudaría a extender la cobertura del aseguramiento en salud.

Las Cajas de Compensación Familiar. Son promovidas activamente por el gobierno nacional para administrar algunos programas públicos, con el argumento de aprovechar su experiencia administrativa y su buena acogida entre la población, ante la cual mantienen una imagen de transparencia y eficiencia. En esa medida, su influencia en la toma de decisiones relacionadas con la protección social en salud es bastante importante.

Su participación fue bastante activa, con permanente disposición al diálogo con los demás actores. A pesar de que su misión institucional se orienta al trabajador activo y su familia, consideran que la creación del Fondo permitiría potenciar su gestión social, contribuyendo a preservar la calidad de vida de los trabajadores durante los períodos de cesantía.

Los empleadores. Constituye un actor sólidamente solidario con las políticas del actual gobierno nacional, al que atribuyen un desempeño destacado en el fortalecimiento del aparato productivo del país. En consecuencia, su apertura a la discusión de propuestas que no provengan del mismo gobierno es bastante limitada. De allí que no se han constituido en un actor proactivo con respecto a la propuesta de creación del Fondo para garantizar el aseguramiento en salud de los TC.

Aunque reconocen que la prioridad del gobierno nacional y local es atender la problemática del desempleo, insisten en que la protección social del TC debe hacer parte de esa agenda prioritaria. Argumentan su postura en el compromiso que tienen con las metas del milenio para combatir la pobreza como parte de su responsabilidad social. Sus opiniones y actitudes de apoyo a la propuesta están condicionadas a estos aspectos: a. Que sea una política pública del Municipio y que el Alcalde sea quien convoque su participación; b. Que no se les exija nuevos aportes económicos y que de hacerlo, se les ofrezca a cambio incentivos de disminución de impuestos. 
Las organizaciones sindicales. Su participación en este proceso fue bastante activa y propositiva. Han mostrado alta receptividad de la propuesta, expresando la necesidad de que sea apoyada tanto por entidades públicas del nivel local, como por otros actores estratégicos de la ciudad.

Consideran que una debilidad de nuestra sociedad es la falta de agremiación y movilización social que representen los intereses de grupos poblacionales. Juzgan que para que los resultados de las investigaciones académicas sirvan para la formación de políticas públicas es necesario que los académicos desborden el límite académico tradicional y avancen hasta el involucramiento en procesos organizativos de los socios comprometidos.

El grupo de investigación. Constituido por investigadores académicos y por decisores públicos del sector de la protección social del Municipio, ha sido el principal promotor de la iniciativa de una política pública para garantizar la protección social en salud de los TC.

Su base conceptual de consenso es que la exclusión del aseguramiento en salud de cualquier sector social es injusta y que la administración municipal debe esforzarse en encontrar estrategias viables que permitan su superación. Desde esa perspectiva, asume el concepto de que el trabajo conjunto entre investigadores académicos y decisores públicos permite mejores alineamientos de las evidencias científicas derivadas de las investigaciones con los procesos políticos propios de la toma de decisiones (19-22).

El mantenimiento de la discusión de la propuesta de intervención se ha facilitado por el interés de los decisores públicos locales y por su posicionamiento político-estratégico, que les otorga gran poder de convocatoria de otros actores. Sin su tramitación burocrática no es posible que la propuesta sea convertida en política de la municipalidad.

Los medios de comunicación. Su involucramiento con la propuesta deviene de la importancia que la opinión pública, a través de las encuestas, le atribuye al tema del desempleo. Su participación se ha concentrado en la facilitación de la interacción entre los investigadores con los TC y con la comunidad en general, a través de la difusión de información sobre las actividades y la propuesta del grupo de investigación. 
Puntos nodales y normas de actuación

Una mirada de conjunto al proceso de discusión de la propuesta permite establecer que los puntos nodales en los que se ha concentrado la interacción de los actores son tres: i. Los espacios de discusión habilitados por el grupo de investigación; ii. El espacio de relacionamiento directo del grupo de investigación con funcionarios de decisión financiera de la administración municipal; iii. El Concejo municipal de Medellín.

Los espacios de discusión habilitados por el grupo de investigación han sido talleres con todos los actores, utilización de espacios en medios de comunicación y eventos académicos. Su activación es ocasional y la participación no está ceñida a ningún marco normativo formal. Han dado lugar a intercambio de opiniones de los actores en relación con la propuesta. En ellos ha prevalecido la voluntad de colaboración, pero igualmente se han expresado los condicionamientos financieros de los empleadores y la exigencia de los decisores públicos municipales del estricto sometimiento a los marcos normativos del SGSSS. Estos espacios también han permitido un acercamiento de los actores para discutir los resultados de la investigación que soporta la propuesta de política pública, propiciando un mejor conocimiento de las condiciones de vulnerabilidad socioeconómica y de aseguramiento en salud de los TC y permitiendo balancear el compromiso de cada actor al respecto.

El espacio de relacionamiento del grupo de investigación con decisores del área financiera de la municipalidad surgió por iniciativa de la Alcaldía municipal, como un espacio de modulación técnica y financiera de la política pública propuesta. También tiene la característica de activarse ocasionalmente. La discusión fundamental ha estado relacionada con la sostenibilidad financiera de la política y su correspondencia con las normas que rigen el gasto social del municipio. No se ha producido todavía un acuerdo definitivo que concluya con el aval financiero de la administración municipal a la propuesta, condición necesaria dentro del marco normativo para que el Concejo Municipal pueda dar curso a su discusión.

El otro punto nodal significativo es el Concejo Municipal, que es el cuerpo de representación política del municipio. Esta corporación tiene una funcionalidad rigurosamente normalizada y le corresponde discutir y aprobar el plan de desarrollo y los programas y/o proyectos que la administración municipal proponga. Hace parte del circuito de decisiones necesarias para formalizar la política pública. Esta circunstancia lo constituye en un actor 
estratégico para su aprobación. Como es usual en este tipo de corporaciones, el nivel de compromiso frente a la propuesta ha sido diferencial entre sus miembros, en función de sus diferentes posicionamientos ideológicos y políticos. Sin embargo, la preocupación que produce el alto nivel de desempleo de la ciudad, la demanda ciudadana de responder a sus consecuencias y el liderazgo de algunos de los concejales, han permitido que se haya mantenido activa la discusión de la propuesta y que haya sido aceptada para incluirse en su agenda de debates.

El complejo de relaciones de los actores interesados y de los actores estratégicos en estos puntos nodales ha producido un consenso tácito (promovido por los decisores públicos, los empleadores y los actores políticos) en que una condición indispensable para mantener la participación de todos ellos en el impulso a la política pública propuesta, es que sea la Administración Municipal la que lidere el proceso y que todas las actuaciones se ajusten a la normatividad del SGSSS.

Esta norma de actuación está sustentada por los actores estratégicos en la necesidad de tener en cuenta la limitación de recursos financieros disponibles para la protección social en salud. Sin embargo, esa norma también desvela el fuerte consenso de los actores estratégicos alrededor de las políticas del gobierno nacional en la materia y su poca disposición a aceptar discusiones significativas sobre las limitaciones del sistema de protección social. Este respeto absoluto de la institucionalidad vigente y de sus mecanismos regulatorios, como norma de actuación que debe cobijar todo el proceso de discusión de la política pública propuesta, ha constreñido los límites en que ella debe moverse y ha excluido la emergencia de alternativas más retadoras para el aseguramiento en salud.

La asimetría de poder de los actores estratégicos con respecto a otros actores participantes que tienen miradas diferentes sobre el SGSSS, ha silenciado la conflictividad latente en sus diferentes puntos de vista.

Procesos de cambio

Entre los alcances que pueden destacarse como resultado de la interacción de los actores involucrados en la propuesta de política pública propuesta por el grupo de investigación, están los siguientes: 
Se ha visibilizado y puesto en la agenda política del gobierno local el problema del no aseguramiento en salud de los TC. En este cambio de condición de un problema público desde su inexistencia como terreno conflictivo a su emergencia como asunto de debate importante para actores diversos, han tenido un rol destacado los investigadores académicos.

Alrededor de la propuesta se surtió un proceso de cambio en el relacionamiento de los actores sociales involucrados, caracterizado por su mayor densidad y su concentración en el diseño de alternativas de política pública para la intervención de esta problemática.

La incorporación de la propuesta en el proceso formal de discusión del Concejo Municipal ha acercado las posibilidades de convertirse en una política pública de la Ciudad. La orientación que tome la decisión será dirimida en el marco del juego de posiciones conceptuales, intereses de los actores y condiciones de asimetría de poder entre ellos.

Se ha propiciado una dinámica inicial de empoderamiento de los TC, que tradicionalmente han sido desatendidos e invisibilizados por los procesos de definición de políticas públicas en la ciudad. El estímulo de los investigadores a la agremiación de los TC ha introducido una discusión más general sobre la plausibilidad de la intervención de los académicos en los procesos sociales de organización. Ha convocado igualmente a la discusión sobre la relación entre los valores éticos y políticos de los investigadores y los procesos de gobernanza en los que se involucran.

\section{DISCUSIÓN Y CONCLUSIONES}

El enfoque analítico de gobernanza propuesto por Hufty permitió destacar instancias importantes de la interacción de actores sociales, que de otra manera pudieron pasar inadvertidas o débilmente consideradas. Sus categorías analíticas parecen ser capaces de capturar, de mejor forma que otras metodologías, la trama de factores que confluyen en los procesos de participación social en asuntos públicos relacionados con la salud.

Una de las mayores fortalezas de esta metodología parece ser la posibilidad que ofrece de apreciar cual o cuales nodos de interacción son potencialmente más dinámicos en la perspectiva de lograr una determinada decisión de política pública, que en la metodología propuesta se asimila a una variable dependiente. La metodología permite dotar al investigador de una panorámica de los 
procesos de interacción social, que facilita extraer conclusiones y disponer etapas subsiguientes de investigación.

En el caso específico de la propuesta de establecer una política pública que garantice la continuidad del aseguramiento en salud de los TC y sus familias, el análisis de "Gobernanza" permitió una mejor y comprensión de cómo ha cambiado y en qué condición se encuentran las interacciones sociales que fluyen en la brega por la garantía del derecho constitucional al aseguramiento en salud de todos los ciudadanos.

La utilización de este marco analítico de gobernanza deberá ser ponderada en relación con otras metodologías que han hecho parte de la caja de herramientas metodológicas de la Salud Pública. Es el caso de la metodología tradicional de análisis de políticas públicas $(23,24)$, el análisis de movimientos sociales $(25,26)$, el análisis de marco lógico (27) y la más reciente propuesta de Mapeo de Alcances (6).

En la aplicación de este enfoque encontramos dificultades al momento de definir y precisar los puntos nodales virtuales del proceso de interacción de los actores, en contraste con los espacios físicos de interacción que fueron más susceptibles de percibirse, describirse y valorarse.

El ejercicio académico realizado enseña que la definición de políticas para solucionar problemas de salud pública en el mundo de hoy no puede hacerse sin la participación activa (con sus respectivos valores y puntos de vista) de la multiplicidad de actores involucrados en el problema a resolver

Agradecimientos. Al IDRC (International Development Research Centre-Canadá), a la Secretaría de Salud de Medellín y a los Grupos de Investigación "Gestión y Políticas en Salud" y "Salud y Sociedad" de la Facultad Nacional de Salud Pública-Universidad de Antioquia, por su apoyo financiero. A la Doctora Luz María Agudelo, Secretaria de Salud de Medellín, por su apoyo al proyecto. Al Doctor Luis Bernardo Vélez, Concejal de Medellín, por su permanente disposición a discutir los resultados de nuestra investigación y a impulsar una política pública de aseguramiento en salud para los TC en la ciudad. 


\section{REFERENCIAS}

1. Plumptre T, Grahan J [Internet]. Governance and Good Governance: International and Aboriginal Perspectives. Canada: Institute On Governance; 1999. Disponible en: http://www.iog.ca/publications/govgoodgov.pdf. Consultado marzo de 2007.

2. Hufty M, Báscolo E, Bazzani R. Gobernanza en salud: un aporte conceptual y analítico para la investigación. Cad. Saúde Pública. 2006; 22 Sup: S35-S45.

3. Bernard, N. A 'New Governance' Approach to Economic, Social and Cultural Rights in the EU. En: Hervey, T.K.; Kenner, J. Economic and Social Rights under the EU Charter of Fundamental Rights: a Legal Perspective. Hart Publishing: Oxford - Portland Oregon. 2003. p. 247-268.

4. Cardona A, Nieto E, Restrepo R, Sierra O, Cárdenas C, Aguirre F. Una propuesta de innovación en políticas de aseguramiento en salud, basada en hallazgos de investigación: el caso de los trabajadores cesantes en Medellín (Colombia). Cad. Saúde Pública, Rio de Janeiro, 2010.

5. Cardona A, Nieto E, Restrepo R, Sierra O, Castaño E, Guarín G. Caracterización de los trabajadores cesantes en Colombia en el periodo 1992-2002, como fundamento para el diseño de una estrategia organizativa y financiera para la sostenibilidad de su aseguramiento en salud. Revista Facultad Nacional de Salud Pública. 2004; 22 (2): 21-34.

6. Earl S, Carden F, Smutylo T. Mapeo de Alcances. Incorporando aprendizaje y reflexión en programas de desarrollo. Libro Universitario Regional (LUR) y el Centro Internacional de Investigaciones para el Desarrollo IDRC. Canadá; 2002.

7. República de Colombia. Congreso de la República. Ley 100 de 1993. Por la cual se crea el Sistema General de Seguridad Social Integral en Colombia.

8. República de Colombia. Ministerio de Salud. Resolución 5261 de 1994. Por la cual se establece el Manual de Actividades, Intervenciones y Procedimientos del Plan Obligatorio de Salud en el Sistema General de Seguridad Social en Salud. El Ministerio, Bogotá; 1994.

9. República de Colombia. Ministerio de la Protección Social. Consejo Nacional de Seguridad Social en Salud. Acuerdo 267 de 2004 por el cual se autoriza la asignación de recursos de la subcuenta de solidaridad del Fosyga para ampliar cobertura en el Régimen Subsidiado de Salud mediante subsidios parciales. Bogotá; 2004.

10. República de Colombia. Ministerio de la Protección Social. Consejo Nacional de Seguridad Social en Salud. Acuerdo 306 de 2005 por medio del cual se define el Plan Obligatorio de Salud del Régimen Subsidiado. Bogotá; 2005.

11. República de Colombia. Ministerio de la Protección Social. Informe de Rendición de Cuentas. El Ministerio. Bogotá; 2008.

12. República de Colombia. Ley 715 de 2001 por la cual se dictan normas orgánicas en materia de recursos y competencias. Bogotá; 2001.

13. República de Colombia. Departamento Administrativo Nacional de Estadística (DANE). Cuentas Nacionales de Colombia. Consolidados de 1996 a 2002.

14. República de Colombia. Departamento Administrativo Nacional de Estadística (DANE). Encuesta Nacional de Hogares; junio de 2000.

15. República de Colombia. Departamento Administrativo Nacional de Estadística (DANE). Encuesta Continua de Hogares; junio de 2004.

16. República de Colombia. Departamento Administrativo Nacional de Estadística (DANE). Gran Encuesta Integrada de Hogares; junio de 2007.

17. República de Colombia. Departamento Administrativo Nacional de Estadística (DANE). Cuentas Nacionales de Colombia; consolidados de 2003 a 2008.

18. República de Colombia. Departamento Administrativo Nacional de Estadística (DANE). Gran Encuesta Integrada de Hogares; mayo de 2009. 
19. International Development Research Centre/Coalition for Global Health Research/Institute of Population Health. Knowledge translation in health and development: research to policy strategies. Ottawa: International Development Research Centre; 2003.

20. Tangcharoensathien V, Wibulpholprasert S, Nitayaramphong S. Knowledge-based changes to health systems: the Thai experience in policy development. Bull World Health Organ 2004; 82:750-6

21. Greenhalgh T, Glenn R, MacFarlane F, Pate P, Kyriakidow O. Diffusion of innovations in service organizations: systematic review and recommendations. Milbank Q 2004; 82:581-629.

22. Brofman N, Langer A, Trostle J. De la investigación en salud a la política: la difícil traducción. México DF/Santafé de Bogotá: Editorial El Manual Moderno; 2000.

23. Meny I, Thoenig JC. Las Políticas Públicas. Ariel. Primera Edición. Barcelona; 1992.

24. Dahl RA. La democracia y sus críticos. Reis.1992; 79 (97): 347-388.

25. Calle A. El estudio del impacto de los movimientos sociales. Una perspectiva global. Reis. 2007; 120 (07):133-153.

26. Vizer E. Socioanálisis: metodología de investigación, análisis diagnóstico e intervención social. Unirevista. 2006;1 (3):1-15.

27. Banco Interamericano de Desarrollo [Internet]. Matriz del Marco Lógico. Una herramienta de gestión para mejorar el desempeño de los proyectos. 1997. Disponible en http:/ /www.iadb.org/cont/evo/spbook/lamatriz.htm Consultado: junio de 2008. 